\title{
COMPORTAMIENTO MECÁNICO DE MATERIAL COMPUESTO REFORZADO CON FIBRAS DE CHONTADURO (BACTRIS GAPISAPES)
}

\section{Mechanical behavior of chontaduro (bactris gapisapes) fiber reinforced material composite}

\author{
Juan Pablo Villate Diaz ${ }^{1}$, Nelson Joman Vargas Ortiz ${ }^{1}$ \\ ${ }^{1}$ MSc. Fundación Universitaria Agraria de Colombia (Bogotá,Colombia).villate.juan@uniagraria.edu.co \\ vargas.nelson@uniagraria.edu.co
}

(Recibido febrero 9 de 2018 y aceptado junio 15 de 2018)

\begin{abstract}
Resumen
Este trabajo presenta los resultados de un programa experimental del comportamiento mecánico de un material compuesto a base de cemento reforzado con fibras de chontaduro. Esta palma (Bactris Gapisapes) es una de las especies de gran abundancia en américa latina y su crecimiento acelerado hace que sea un material accesible para posterior uso. Se realizaron ensayos a tracción directa tanto de la fibra como del compuesto. Para el compuesto se usó una fracción volumétrica de fibra del $4 \%$ extraída del tronco de la palma. La resistencia a tracción de la fibra resultó en valores de 190 MPa y módulo de elasticidad de 7,3 GPa. El compuesto reforzado con la fibra de chontaduro resulto con la formación una única fisura con una resistencia a tracción de $3 \mathrm{MPa}$, estos resultados servirán para generar un mejoramiento en el comportamiento mecánico y poder generar un material adecuado para ser usado en construcciones rurales en elementos de muros de carga.
\end{abstract}

Palabras clave: fibras de chontaduro A; Compuesto a base de cemento B; Comportamiento mecánico C.

\begin{abstract}
This work presents the results of an experimental program on the mechanical behavior of chontaduro palm fibers and cement based material composites reinforced with these fibers. This palm (Bactris Gapisapes) is one of the species of great abundance in Latin America and its accelerated growth makes it an accessible material for later use. Additionally, the fibers were used as reinforcement in cement based composites using volumetric fractions of $4 \%$ extracted from the trunk of the palm. The tensile strength of the studied fibers varied of $190 \mathrm{MPa}$ and the modulus of elasticity of 7,3 GPa. The composites presented a strain softening behavior with the formation of only one crack. The tensile strength of the composite of $3 \mathrm{MPa}$. these results will serve to generate an improvement in the mechanical behavior and to be able to generate a suitable material to be used in rural constructions in load-bearing walls elements
\end{abstract}

Keywords: chontaduro fibers A; Cementitious composite B; Mechanical behavior C.

\section{INTRODUCCIÓN}

Las técnicas usadas tradicionalmente para la construcción son evaluadas por normas y métodos de diseño en la ingeniería, las cuales se van actualizando diariamente para optimizar las propiedades fisico,- mecánicos de los materiales. Sin embargo, el uso de materiales naturales está limitado por no contar con estudios necesarios y técnicas de conservación de los mismos y aún más por no contar con normatividad para su utilización en construcciones civiles, puesto que normas como el reglamento colombiano de construcción sismo resistente (NSR-2010), solo tienen parametrizados el uso de materiales no convencionales como la guadua y la madera, esto se ve reflejado en que las comunidades rurales no hacen uso de los materiales autóctonos para la construcción, mejoramiento y adecuación de sus viviendas. Por eso hace tan importante el estudio de sus propiedades mecánicas con el fin de que su uso no sea empírico, sino al contrario se tengan unos parámetros claros del comportamiento real y metodológico como ejemplo el material estudiado en este trabajo. Los 
materiales de construcción convencionales como el acero y concreto, son los componentes más relevantes en la infraestructura y presentan una alta demanda hoy en día, aunque por los elevados costos de estos materiales y gran el impacto negativo en el medio ambiente, se han aumentado el uso de materiales sustentables como son los compuestos reforzados con fibras naturales [1]. Estos compuestos naturales pueden reducir el desecho de la industria de la fabricación del material, adicional a esto aumenta la eficiencia energética al ofrecer una solución a la infraestructura en poblaciones menos favorecidas, así como promover el concepto de sustentabilidad.

Estudios sobre compuestos reforzados con fibras largas y bidireccionales, muestran que tienen una a mayor resistencia a la tracción y una capacidad de absorción de energía alta con la formación de múltiples fisuras. [8] [9]. Para esto el contenido de fibra debe ser mayor que la fracción crítica. [10]. Los estudios en la interfaz de la fibra natural con una matriz de cemento muestran que las secciones transversales irregulares presentan altos niveles de adherencia, con valores promedio hasta de $0.92 \mathrm{MPa}$ (para sisal fibras). [11]. En el caso de que se produzca una alteración en la interfaz, por ejemplo, con un recubrimiento polimérico estos valores de adherencia pueden mejorar y aumentar hasta $1.25 \mathrm{MPa}$ [8].

La búsqueda de nuevos materiales sustentables, han motivado este trabajo. Los autores han buscado una alternativa con la una fibra natural como la de chontaduro, además que esta planta tiene un crecimiento rápido. El objetivo primordial del presente trabajo fue evaluar el comportamiento mecánico a tracción directa tanto de la fibra como del compuesto a base de cemento reforzado con fibras de chontaduro. [4] [12] [3].

\section{METODOLOGÍA}

Se obtuvo una palma con una edad de 2 años en Buenaventura - Colombia (ver figura 1) y que presentaran una coloración uniforme y que además permitieran observar si estas se encontraban sanas, es decir que permitieran observar que estas no presentaran fisuras, golpes o perforaciones bien sea por la intemperie o por plagas de insectos.
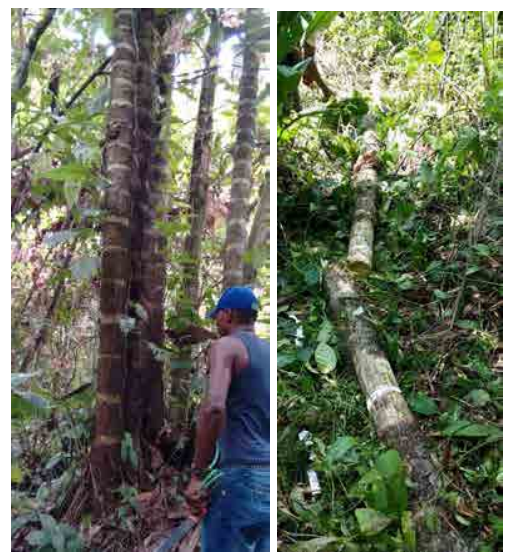

Figura 1. Palma de chontaduro

De las muestras de chontaduro se extrajeron las fibras con diámetros de $0.5 \mathrm{~mm}$ y 1 metro de longitud, esta extracción se puede realizar de dos maneras, la primera por medio de un trapiche, el cual es un molino mecánico que aplasta la el tallo dejando expuesta su parte interna, y la segunda es de forma manual, la cual se realiza por medio de un martillo que fractura la capa externa de la palma y manualmente se extraen las fibras de las capas internas (ver figura 2). Para este estudio se eligió realizar este procedimiento de forma manual ya que era menos agresivo con la muestra puesto que al soportar la carga del molino, podría verse afectada la resistencia posterior de las fibras.

(a)

(b)

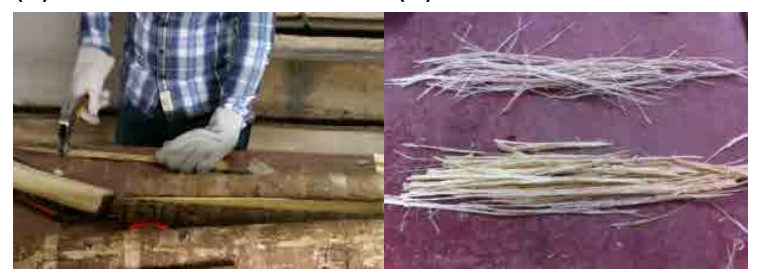

(c)

(d)

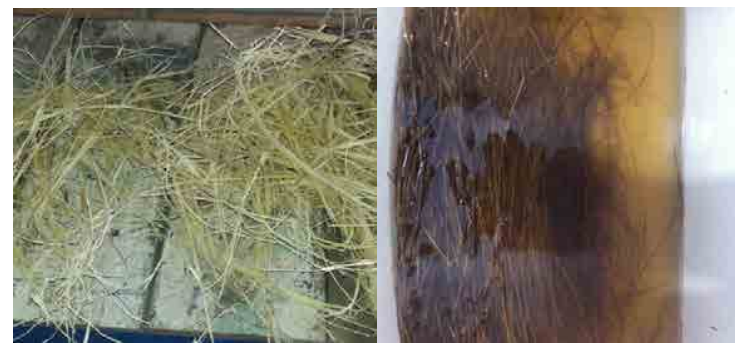

Figura 2. Extracción manual de fibras de chontaduro. (a) extracción manual con martillo. (b) fibras extraídas. (c) tratamiento con agua a temperatura de $30 \stackrel{\circ}{\circ}$ y (d) secado a intemperie. 
Luego de extraer las fibras, se someten a un lavado con agua a una temperatura de $30^{\circ} \mathrm{C}$, para después dejar que estas se secaran a una temperatura de $28^{\circ} \mathrm{C}$ (ver figura 2), ya que la humedad interna que traen al estar en contacto con el ambiente exterior genera que en las fibras se presenten hongos, los cuales afectan de manera notable la resistencia y por tanto los resultados de esta investigación. Al tener una buena cantidad de fibras, se realizó una segunda selección más específica y rigurosa, en la cual se escogieron fibras que tuvieran un diámetro calidad similar de $0.5 \mathrm{~mm}$ para tener unos resultados más confiables.

Las fibras de chontaduro se organizaron unidireccionalmente, en el sentido axial a la carga para que los esfuerzos a tracción estuvieran distribuidos al largo de la fibra (ver figura 3). Los ensayos a tracción directa de materiales compuestos a base de cemento usando fibras no tiene una normalización clara, puesto existen muchos factores que pueden varia su resultando, como la fragilidad del concreto, la iteración entre las el sistema de agarre entre el cuero de prueba y la maquina universal, en ese caso se siguió la normativa ASTM C 150-07 y ASTM C 1557, las cuales siguen parámetros de especificaciones estándares para cemento portland y métodos de ensayo directo para determinar módulo de elasticidad de fibras.

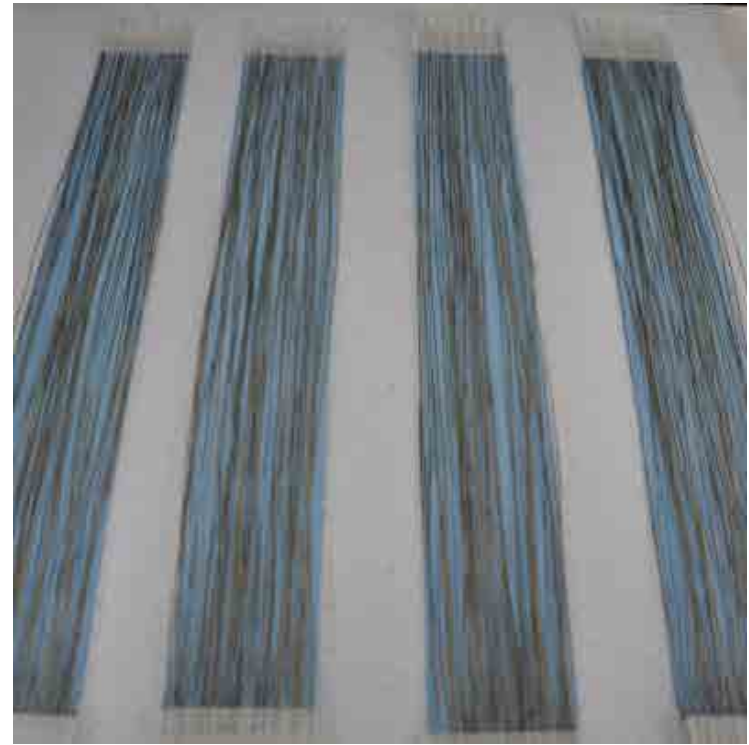

Figura 3. Fibras organizadas para colocar embebidas unidireccionalmente al compuesto
Los ensayos de tracción directa del material compuesto, donde su matriz fue realizada con un mortero con una dosificación en peso de 1:1:0,4 (cemento: arena de peña: agua/cemento), se realizó en el modelo MTS 311 con una celda de $1000 \mathrm{kN}$. La velocidad de desplazamiento fue $0,5 \mathrm{~mm} / \mathrm{min}$ (ver figura 4). Las dimensiones de cada cuerpo de prueba fueron $400 \times 50 \times 15$ $\mathrm{mm}$ (longitud $\mathrm{x}$ ancho $\mathrm{x}$ espesor), se reforzaron con fibras de chontaduro unidireccionalmente con 4 capas de fibras sin tratamiento, que es equivalente a un volumen de $4 \%$ respectivamente, esta cuántica se determinó según el número de fibras obtenido.
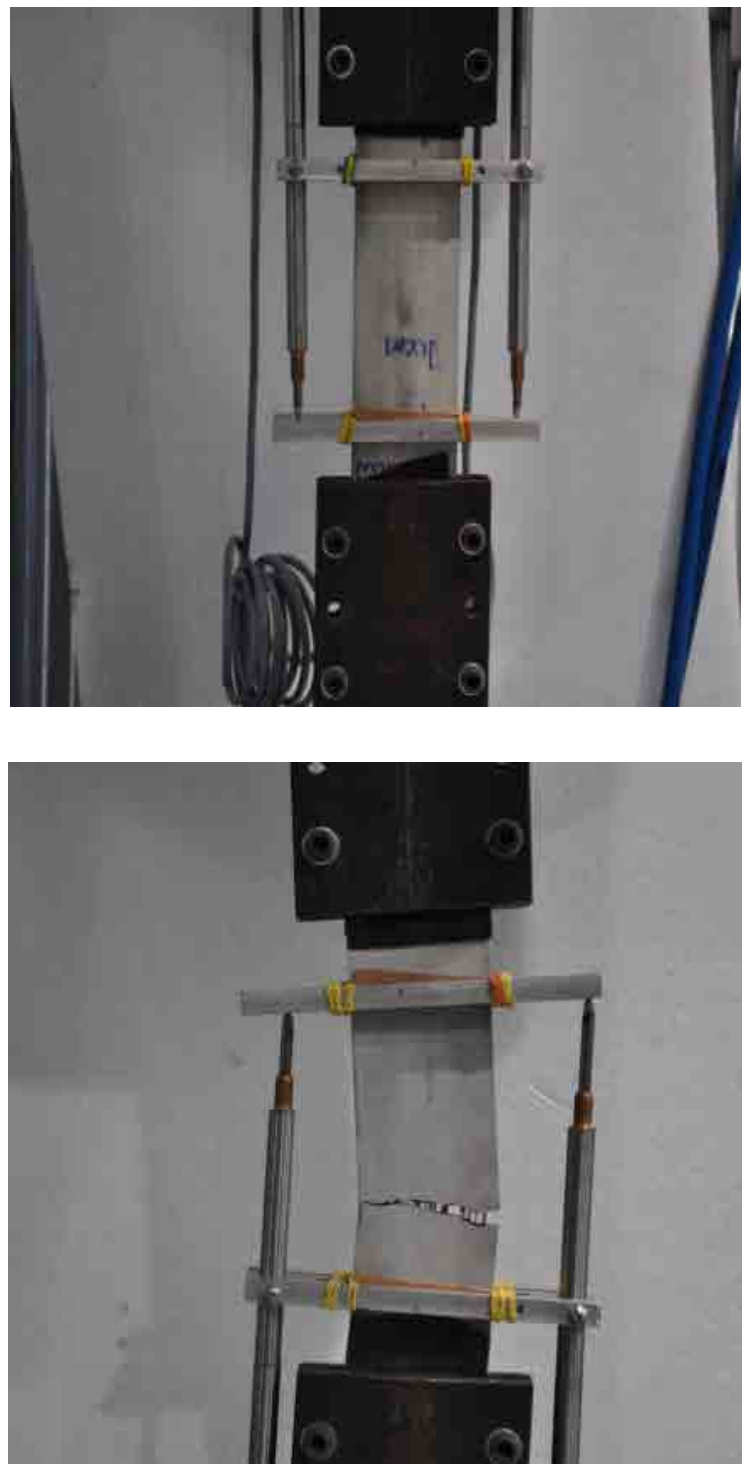

Figura 4. Ensayo de tracción directa del material compuesto

Rev. Ing. Investig. Desarro. Vol.18 (2). pp. 29-34, Julio-Diciembre. 2018, Sogamoso-Boyacá, Colombia. 
El ensayo de adherencia se realizó con tubos de PVC de diámetro de $2 \mathrm{~cm}$ y longitudes de fibras embebidas de 5 $\mathrm{cm}$, donde la maquina universal MTS, realizaba un proceso de arrancamiento de la fibra.

\section{RESULTADOS}

La fibra de chontaduro obtuvo un módulo de elasticidad de $8 \mathrm{GPa}$ y una resistencia a tracción de $190 \mathrm{MPa}$ (ver tabla 1), donde comprado con otras fibras vegetales, muestra que la resistencia a tracción directa de esta fibra es baja, siendo más resistentes fibras de coco (192 MPa), fibra de sisal (546 MPa) y fibras de bambú (812 MPa) [2] [3] [4] [5]. Este comportamiento influye en los resultados del material compuesto, por lo tanto, su comportamiento junto a su superficie lisa (sin estrías o corrugaciones que mejoren el comportamiento de adherencia fibra- matriz) y la baja fracción volumétrica de la fibra (4\%), puede ser determinante para su comportamiento son la formación de una sola fisura.

Tabla 1. Resultados de ensayo a tracción directa de fibras de chontaduro.

\begin{tabular}{|c|c|c|c|c|c|}
\hline \multicolumn{2}{|c|}{$\begin{array}{c}\text { Resistencia } \\
\text { máxima a tracción } \\
\text { (MPa) }\end{array}$} & \multicolumn{2}{|c|}{$\begin{array}{c}\text { Deformación } \\
\text { máxima } \\
(\%)\end{array}$} & \multicolumn{2}{|c|}{$\begin{array}{c}\text { Módulo de } \\
\text { elasticidad } \\
\text { GPa }\end{array}$} \\
\hline 190 & \pm 17.8 & 2.8 & \pm 0.3 & 7,3 & \pm 0.8 \\
\hline
\end{tabular}

La resistencia máxima de adherencia a la tracción de la matriz de cemento y las fibras de chontaduro, calculada a partir de la ecuación (1) es de 0,15MPa (ver tabla 2). Otras fibras naturales tales como la fibra de sisal presentan valores de $0.92 \mathrm{MPa}$ [11] y fibras de juta presentan valores de $1.25 \mathrm{MPa}$ [8]. Los resultados indican que la adherencia de la fibra con la matriz es débil, comparados con otras fibras. La resistencia de adherencia ( $($ nom). [14] [11].

$$
\mathrm{t}_{\text {nom }}=\frac{P_{\max }}{\mathrm{pf} L}
$$

Donde Pmax es la fuerza máxima de adherencia, $L$ es la dimensión de inserción y es el de la medición del diámetro.

Tabla 2. Resultados ensayo de adherencia fibra-matriz.

\begin{tabular}{|c|c|c|c|}
\hline $\begin{array}{l}\text { Longitud de } \\
\text { recubrimiento } \\
\text { (mm) }\end{array}$ & $\begin{array}{l}\text { Pmax } \\
\text { (N) }\end{array}$ & $\begin{array}{c}\delta \\
\text { (correspondiente } \\
\text { a Pmax) } \\
(\mathrm{mm})\end{array}$ & $\begin{array}{l}\text { tnom } \\
\text { (MPa) }\end{array}$ \\
\hline 80 & $27 \pm 6.9$ & $2.3 \pm 1.7$ & $0.15 \pm 0.04$ \\
\hline
\end{tabular}

Una de las consecuencias de no obtener un comportamiento dúctil conformación de múltiples fisuras, es la baja adherencia de la fibra de chontaduro con la matriz de cemento, que también se mencionó en otros estudios [15]. Se puede ver que el comportamiento mecánico del compuesto, cuando se utiliza fibras de chontaduro, la resistencia a tracción aumenta hasta una resistencia máxima de 3,05 MPa (ver tabla 3 y 4), aunque se presente una formación de una sola fisura (ver figura.5), después existe un endurecimiento causada por la resistencia establecida por las fibras, hasta la falla de estas. Este comportamiento frágil, puede ser debido también a la baja fracción volumétrica de fibra en la matriz.

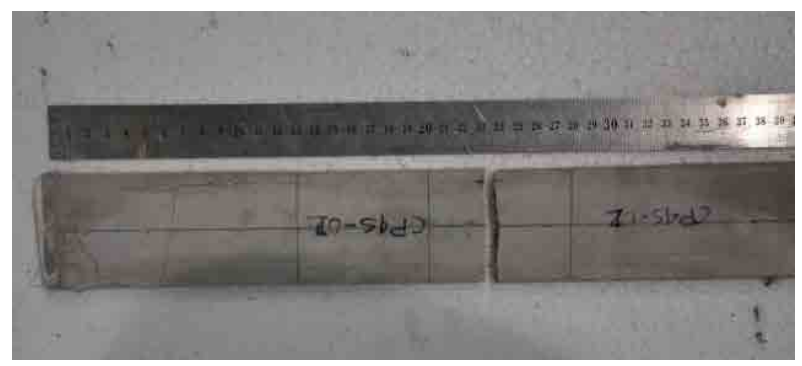

Figura 5. Formación de única fisura dentro del rango del compuesto

Tabla 3. Resultados ensayo a tracción directa de compuesto a base de cemento reforzado con fibras de chontaduro (resistencia máxima a tracción).

\begin{tabular}{|c|c|c|c|}
\hline \multirow{2}{*}{ Compuesto } & \multirow{2}{*}{$\begin{array}{l}\text { Fracción } \\
\text { volumétrica de } \\
\text { fibra } \\
\text { (\%) }\end{array}$} & \multicolumn{2}{|c|}{$\begin{array}{c}\text { Resistencia máxima } \\
\text { a tracción }\end{array}$} \\
\hline & & & (MPa) \\
\hline Matriz & - & 1.5 & \pm 0.12 \\
\hline 4 capas de fibras & 4 & 3.05 & \pm 0.52 \\
\hline
\end{tabular}


Tabla 4. Resultados ensayo a tracción directa de compuesto a base de cemento reforzado con fibras de chontaduro (deformación máxima).

\begin{tabular}{cccc}
\hline Compuesto & $\begin{array}{c}\text { Fracción } \\
\text { volumétrica de } \\
\text { fibra } \\
\text { (\%) }\end{array}$ & $\begin{array}{c}\text { Deformación } \\
\text { máxima }\end{array}$ \\
\hline Matriz & - & 0.01 & \pm 0.001 \\
4 capas de fibras & 4 & 0.3 & \pm 0.06 \\
\hline
\end{tabular}

\section{CONCLUSIONES}

La matriz mejoró su comportamiento mecánico con la adición de fibras de la palma de chontaduro, pero se debe mejorar la interface fibra - matriz para mejorar este comportamiento de formación de múltiples fisuras a tracción directa.

La fibra presenta una superficie muy lisa, lo cual se necesita un tratamiento en la misma para mejora la adherencia fibra - matriz.

La matriz presentó un buen comportamiento a compresión, no obstante, a tracción presentó un comportamiento deficiente por esto se analizó el compuesto solo a tracción.

El comportamiento mecánico del material compuesto no es el adecuado para seguir con la etapa de generación de un nuevo material, esto es debido a su bajo porcentaje de fibra usada en el compuesto; la adherencia pobre entre fibra y matriz, por tal motivo es importante mejorar los aspectos anteriormente mencionado para adecuar mejor el material a las solicitaciones evaluadas.

\section{RECOMENDACIONES PARA FUTUROS TRABAJOS}

Ensayos de pull-out para determinar interface fibra matriz relacionando ensayos de microscopia electrónica por barredura para determinar morfología de fibra e incidencia de vacíos en el comportamiento mecánico de la fibra, mejoramiento de superficie de fibra para determinar mejoramiento en comportamiento mecánico de compuesto, ensayos de durabilidad para determinar degradación de fibra.
Ensayos con otras fibras (caña brava, sisal y coco.) para determinar cuál es la fibra más apropiada para el compuesto.

Ensayo de muro de carga escala real para determinar su comportamiento mecánico real.

Modelar y realizar memoria de cálculo de diseño de casa de un piso unifamiliar como sistema constructivo de muros de cargas.

Construcción de casa unifamiliar de un piso según todo el estudio realizado anteriormente.

\section{REFERENCIAS}

[1] J. George, M. S. Sreekala, S. Thomas, "A Review on Interface Modification and Characterization", Revista 41Polymer engineering and science, vol. 41. $\mathrm{n}$ o 9, pp 1471-1485, noviembre 2015.

[2] D. B. Dittenber, H. V. S. GangaRao, "Critical review of recent publications on use of natural composites in infrastructure", Revista Composites Part A: Applied Science and Manufacturing, vol.43 $\mathrm{n} \cong 9$, pp 1419-1429, agosto 2012.

[3] R. D. Tolêdo Filho, K. Ghavami, G. L. England, K. Scrivener, "Development of vegetable fibre-mortar composites of improved durability", Revista Cement and Concrete Composites, vol. 25 n. 2, pp. 25, 185, febrero 2003.

[4] F. d. A. Silva, R. D. T. Filho, J. d. A. M. Filho, E. d. M. R. Fairbairn, "Physical and mechanical properties of durable sisal fiber-cement composites", Revista Construction and Building Materials, vol. 24 n.o 5 pp. 777-785, mayo 2010.

[5] A. Alida, M. N. Mazlee, B. J. Shamsul, H. Kamarudin, "Composite cement reinforced coconut fiber: Physical and mechanical properties and fracture behavior", Australian Journal of Basic and Applied Sciences, vol. 5. Pp 1228-1240, noviembre 2011.

[6] D. G. Swift, R. B. L. Smith, "The flexural strength of cement-based composites using low modulus (sisal) fibres", Revista Composites, vol.10 n.o 3, pp. 145-148, julio 1979.

[7] P. R. Lima Lopes, R. D. Toledo Filho, "Uso de metacaulinita para incremento da durabilidade de compósitos à base de cimento reforçados com fibras de sisal" Revista Ambiente Construido. Vol.8 n. 4. Pp. 7-19, enero 2008. 
[8] M. E. Alves Fidelis, F. de Andrade silva, R. Dias Todelo Filho, "The Influence of Fiber Treatment on the Mechanical Behavior of Jute Textile Reinforced Concrete", Revista Key Engineering Materials, vol. 600. pp 469-474, febrero 2014. DOI: https://doi. org/10.4028/www.scientific.net/KEM.600.469

[9] F. d. A. Silva, D. Zhu, B. Mobasher, C. Soranakom, R. D. Toledo Filho, "High speed tensile behavior of sisal fiber cement composites", Revista Materials Science and Engineering: vol. 527 n. 3. pp 544552, enero 2010.

[10] A. Bentur, S. Mindess, "Fibre reinforced cementitious composites", CRC Press Ed, 2nd edición. Pág. 624. London, abril 2014.

[11] F. d. A. Silva, B. Mobasher, C. Soranakom, R. D. T. Filho, "Effect of fiber shape and morphology on interfacial bond and cracking behaviors of sisal fiber cement based composites". Revista Cement and Concrete Composites. vol.33 n.o 2. pp 814-823. Septiembre 2011.

[12] R. D. Toledo Filho, F. d. A. Silva, E. M. R. Fairbairn, J. d. A. M. Filho, "Durability of compression molded sisal fiber reinforced mortar laminates" Revista
Construction and Building Materials. vol. 23 n. 6. pp 2409-2420. junio 2009.

[13] B. J. Mohr, J. J. Biernacki, K. E. Kurtis, "Supplementary cementitious materials for mitigating degradation of kraft pulp fiber-cement composites". Revista Cement and Concrete Research, vol. $37 n^{\circ} 11$. pp 1531-1543. noviembre 2007. DOI: https://doi. org/10.1016/j.cemconres.2007.08.001

[14] M. Z. Rong, M. Q. Zhang, Y. Liu, G. C. Yang, H. M. Zeng, "The effect of fiber treatment on the mechanical properties of unidirectional sisal-reinforced epoxy composites". Revista Composites Science and Technology. vol $61 \mathrm{n}^{\circ} 10$. Pp 1437-1447. agosto 2001. DOI: https://doi.org/10.1016/S02663538(01)00046-X

[15] M. A. de Farias, M. Z. Farina, A. P. T. Pezzin, D. A. K. Silva, "Unsaturated polyester composites reinforced with fiber and powder of peach palm: Mechanical characterization and water absorption profile", Revista Materials Science and Engineering: $C$, vol 29 n². pp 510-513. marzo 2009. DOI: https:// doi.org/10.1016/j.msec.2008.09.020 